\title{
Feasibility of Using a Lightweight Context-Aware System for Facilitating Reliable Home Blood Pressure Self-Measurements
}

\author{
K. Kusk, D. B. Nielsen, T. Thylstrup, N.H. Rasmussen, J. Jørvang C. F. Pedersen, S. Wagner \\ Aarhus University, Department of Engineering, \\ Aarhus, Denmark
}

\begin{abstract}
Blood pressure self-measurement requires the patient to follow a range of recommendations in order to be considered valid for diagnostic use. We investigated how a lightweight and context-aware system designed for sensing and correcting incorrect usage of home blood pressure selfmeasurement devices was perceived by a group of four patients. We found that using a context-aware system for improving adherence is feasible for overcoming the challenges related to moving from the supervised clinical setting to the unsupervised home setting. We also found that patients are willing to accept more advanced monitoring systems and increased surveillance, as long as it is only used during selfmeasurement periods and is proportional to the severity of the disease.
\end{abstract}

Keywords; Self-care, patient autonomy, blood pressure selfmeasurement, adherence, reliability, context-aware, pervasive healthcare, telemonitoring, telemedicine, e-Health.

\section{INTRODUCTION}

Blood pressure self-measurement (BPSM) is used in the diagnosis and monitoring of hypertensive patients and other chronic patient groups [1]. BPSM requires the patient to follow a range of recommendations in order to be considered valid for diagnostic use and provide unbiased results [2]. These recommendations include that the patient must be correctly seated, rested for five minutes, not moving or talking, and in a quiet environment before and during measurements [1-3]. However, current state-of-the-art blood pressure (BP) devices cannot verify whether the patient complies or not, and the majority of patients using BPSM do not know of, or do not follow, these recommendations [3]. Previous studies have shown that context-aware technology can be used for detecting operator-error when using a device in the unsupervised setting [4-7]. However, these solutions were designed using intelligent furniture which may not be feasible for everyday use in the general practice clinic as a lend-home service to patients for self-monitoring purposes. As an alternative, we suggest constructing a lightweight system relying on sensor fusion to model the user's context before and during measurements. Such a system should be able to verify whether important context factors of the patient meet the given recommendations, as well as provide the user with interactive and context-aware feedback.
The aim of this study was to investigate how self-care patients would perceive increased surveillance in their home in the shape of a context-aware system. To this purpose, we developed the research prototype BP@Home and evaluated it qualitatively with four hypertensive patients in their home setting.

\section{MethodS AND MATERIALS}

\section{A.BP@Home research prototype}

The BP@Home system uses rule based logic to detect whether the patient observes the recommended rest time, refrains from moving excessively, and is in a quiet surrounding while self-measuring. This is done using two passive infrared movement sensors (Panasonic motion sensor AMN21111) and a microphone-based sensor for measuring the sound level. This approach has previously been described in detail and validated in a laboratory study [8]. BP@Home guides the patient using context-aware aids to correct any non-adherent behavior in order to obtain the most valid BP values, while registering the actual context of the patient, and providing context-tagging of the healthcare data. The system (figure 1) is based on a Continua certified IEEE 11073 compliant BP device (A\&D Medical, UA-767PBT-C) with a Bluetooth interface a custom-build sensor box consisting of a wireless sensor node (Shimmer), with the three sensors, and a tablet computer for user interface (Samsung Galaxy II).

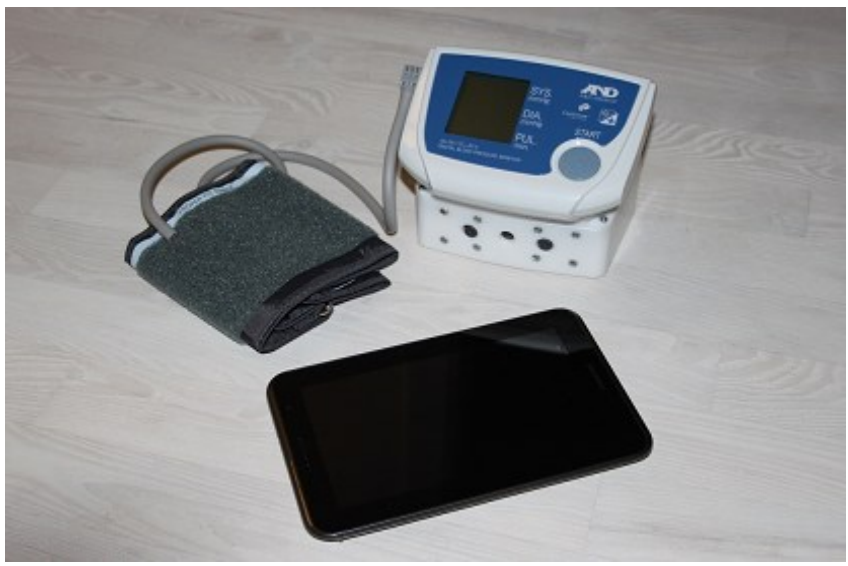

Figure 1. The solution consists of the A\&D blood pressure device, shown on top of the wireless sensor box (above), as well as the Samsung Android tablet computer running the BP@Home application (below). 
The tablet computer communicates with a central database server called Net4Care using the $3 \mathrm{G}$ interface of the tablet computer. Net4Care is a tentative implementation for the national Danish healthcare infrastructure for exchanging biomedical data. The sensor box contains a sound sensor and two infrared movement sensors adapted for an embedded device with a wireless interface for tablet communication. The tablet guides the patient through the self-measurement process (see figure 2).

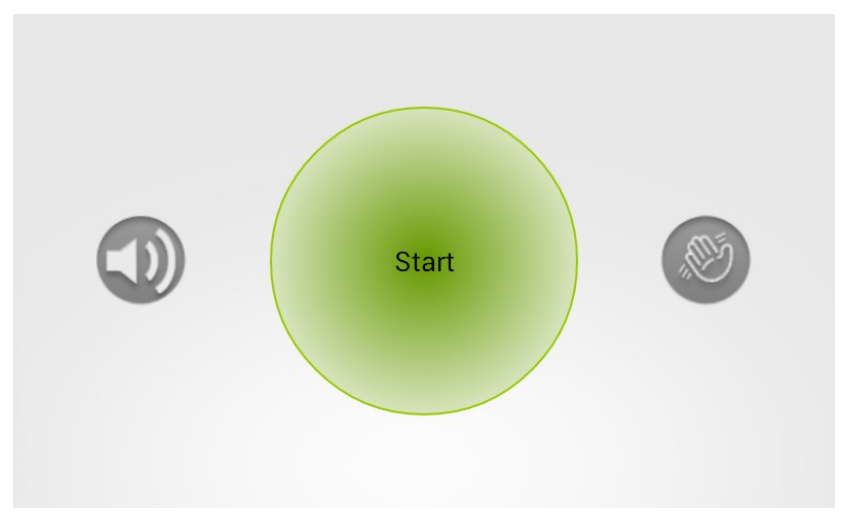

Figure 2. BP@Home start screen

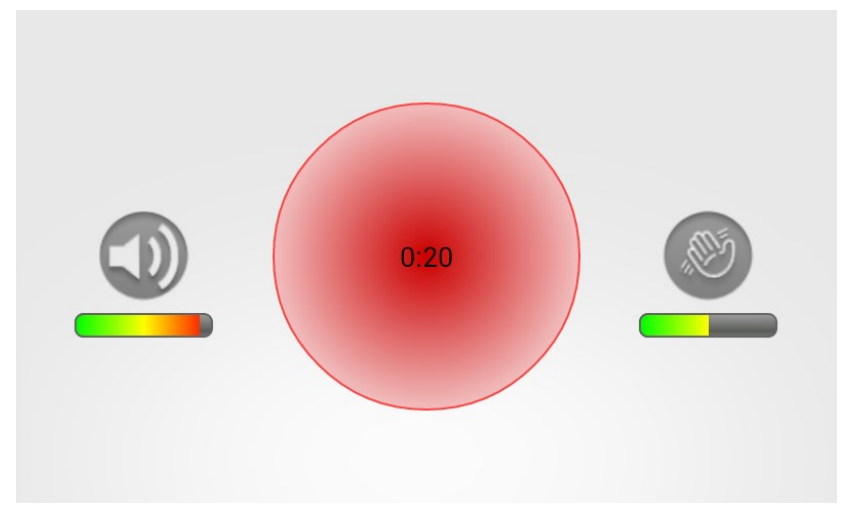

Figure 3. BP@Home countdown and process bars showing the level of movement and sound

After the user presses "Start", a countdown period of 5 minutes is initiated ensuring that the patient observes the five minutes rest period (see figure 3). During the rest period the sensor box senses the context and relays the information to the tablet application. The tablet application shows the accumulated ambient sound and movement levels in two process bars as contextual feedback to the patient.

The BP@Home application is designed to support switching between two different modes of operation representing different levels of patient guidance. The first mode does not guide the user actively, but only records the level of adherence for later verification, while the other mode guides the patient actively. The first mode can then be used during the first three days of self-measurement. Then, BP@home automatically switches to the second mode for the remaining three days, allowing the user to act as selfcontrol.

The study is part of the UNIK partnership project Connect2Care and funded by the Danish Agency for Science, Technology and Innovation, Central Denmark Region, and Region of Southern Denmark.
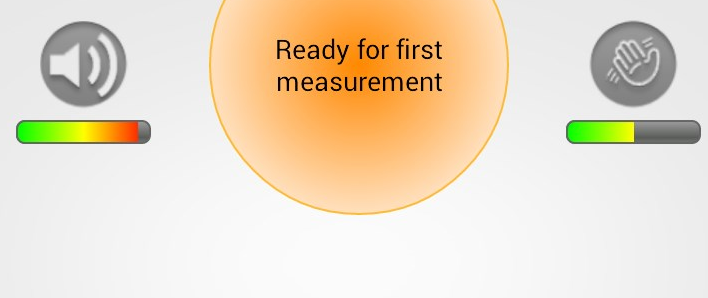

Figure 4. BP@Home has finished the countdown for the rest period and is now ready for the first measurement.

Once the rest period is completed, the patient is guided to start the BP device (see figure 4). Once the measurement is received by the $\mathrm{BP} @$ home application, the user is challenged to either accept or redo the measurement (see figure 5). Finally, the patient is led to a result screen with an overview of the measurements along with an indication of the adherence level of the last measurement (see figure 6). The final color of the two bars represents the context tagging of the measurement. Green is used to indicate acceptable levels, red to indicate unacceptable, while yellow indicates borderline acceptable.

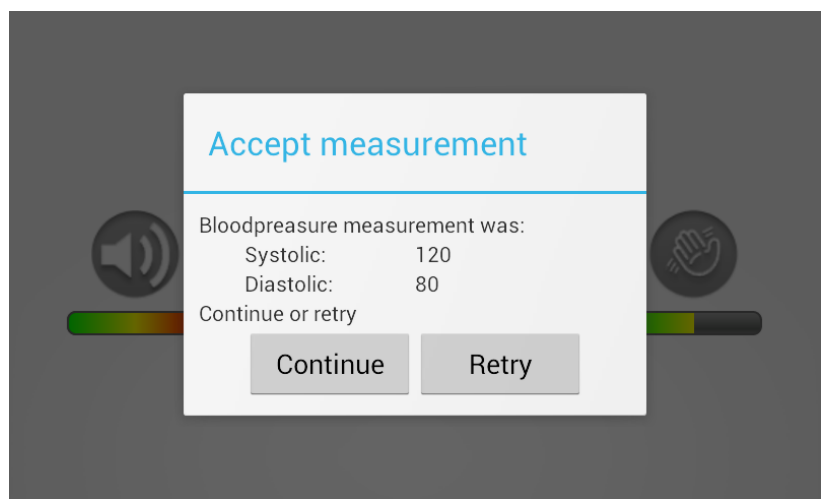

Figure 5. BP@Home receives the blood pressure values. The patient has the choice to continue or redo the measurement.

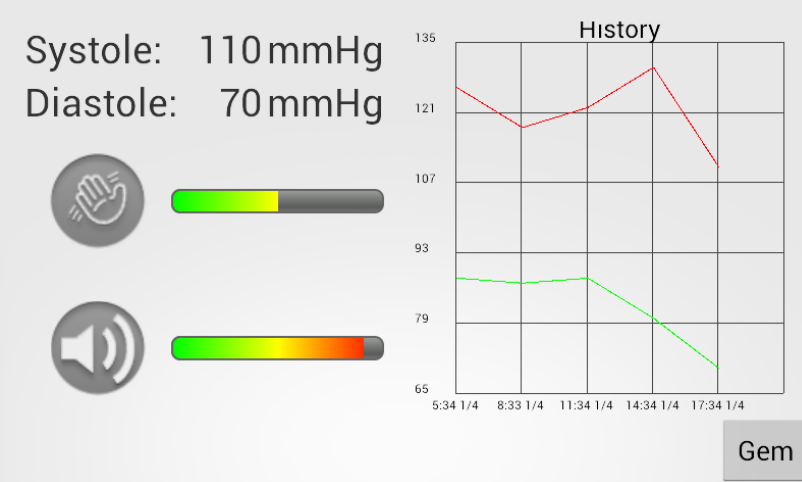

Figure 6. BP measurement values and the level of movement and sound from the five minutes rest period. 


\section{B. Methods}

The BP@Home application was developed following a participatory design approach employing the Adherence Strategy Engineering Framework (ASEF) as the primary design method [7]. Both healthcare professionals and hypertensive patients were involved in the design of the research prototype.

Initially, three semi-structured interviews with three general practitioners (GP) and two nurses, at three general practice clinics were done as a preliminary investigation of state-of-the-art methods and devices currently used for BPSM in the clinical praxis. We wanted to learn which information the patient receives when the home BP measurement device is sent home with the patient, and how GPs evaluate the BP values when the patient returns with the device. Furthermore, the initial prototype was introduced to the GPs and staff members to get their feedback as input for the participatory design process.

Following this, an open-ended interview with a 64 year old male patient utilizing a home measurement device was performed to investigate the patient's perspective on home BPSM. The interview occurred in the home of the patient, enabling him to show how and in which physical context the measurements are done. Furthermore, we observed a selfmeasurement procedure performed by the patient.

We then invited a focus group of five hypertensive senior citizens to a design workshop for a discussion of home monitoring and self-care in general, and BP measurement in particular, and for finalizing the design of the suggested solution.

Following this, four members of the focus group accepted to evaluate the prototype during a six day home measurement evaluation trial. Semi-structured individual interviews were performed before onset of the field trials, and as follow-up interviews.

\section{RESULTS}

We identified the following themes emerging from our design workshop and interview sessions, including before and after the four six day field trials.

\section{A. Previous experiences with self-care technology}

The four participants all stated to have previously used a BP device provided by their GP at home. Thus, they were already familiar with the concepts of self-care and selfmeasuring at home. They all found it very useful to be able to monitor their health and follow the progression of their BP. The test subjects all have access to and own a computer, which is "used on a daily basis" or "frequently", including for emails, online banking, and more. One participant frequently experienced a high level of white coat effect [1-4] when being measured at the clinic, and even previous self-monitoring in the home setting was perceived as stressful.

\section{B. Perceived benefits of self-care technology}

The participants found self-care technology to be a suitable supplement or even alternative to visiting the GP. Two of the participants talked of the benefits of getting a regular status of the disease progression, while all participants found it would be beneficial if visits to the GP's office could become less frequent or even avoided all together. Saving a trip to the doctor was perceived as an important step towards not becoming stigmatized by their chronic diseases, feeling less as a patient, and more as an empowered individual.

\section{Proportionality of surveillance vs. increased adherence}

All test participants initially found that it was fully acceptable if a device corrected them during selfmeasurement in case of operator error. Provided that the aim of the surveillance effort was to achieve increased adherence and higher data quality, and that the device would be able to pinpoint the reason for the error and instruct them how to act instead. The participants felt more secure with a system monitoring their adherence levels. However, the surveillance should only be active during measurements. Specifically, the participants pointed out that they did not want to be video monitored at any time. However, recording audio and movement levels was perceived as acceptable. Furthermore, the level of surveillance should be proportional to the disease. As two of the participants speculated; patients who are only suffering from hypertension might not advocate full surveillance. While in the case of other and more severe conditions, increased surveillance might be justifiable and even desirable.

\section{My home is not the clinic}

Neither of the participants felt the introduction of more self-care technology in the home setting to be an increased invasion-of-privacy. However, it was underlined that technology should be small and storable, in a closet, a drawer, or similar, so it would be easy to hide away when having visitors, as well as when travelling or visiting friends. The participants in general did not like to get reminded of their disease, and did not want to display their disease to others through technology. Again, the severity of the disease vs. the level of technology intervention and adherence strategy used should at all times be proportional.

\section{E. Using a tablet computer as interface}

The participants were generally very pleased with the tablet computer as a user interface. One participant who experienced initial technical problems still found it usable and relevant. The ability to follow the progression of the BP, including retrieving previous measurements was found very usable. It was however pointed out, that the sensor box should be an integral part of either the tablet or the BP device and not a separate device. 


\section{DISCUSSION}

We found that the participants were willing to accept more advanced monitoring systems and increased surveillance, as long as they are only used during selfmeasurement periods and that the surveillance levels are proportional to the severity of the disease. Thus, we suggest that introducing increased context awareness in the shape of adherence verifiers and aids should be considered to augment existing self-care systems and devices. This is in line with previous work in the area [11].

Several related studies have investigated challenges related to BPSM in the home setting. These studies investigated the ability of the patients to correctly report the self-measured data, and found that $16-50 \%$ of all reported data contained errors [12-15]. However, none of the four studies investigated patient adherence to recommendations, but only focused on the accuracy of self-reporting. Two recent studies have investigated self-measurement in the hospital outpatient setting for both pregnant women [16] and renal disease patients [17]. Both studies found that unsupervised measurements are subject to critical bias significantly reducing the data quality. An additional two studies have investigated the potential for using contextaware sensors for guiding patients during 24 hour ambulatory BP measurement $[18,19]$. However, these studies were never tested in the field with patients.

Thus, in order to further investigate these topics we suggest planning a randomized clinical trial in the home setting with a sufficient patient cohort in order to conclude whether our approach is a reliable method as compared to BPSM.

\section{CONCLUSION}

We found it feasible to use a context-aware system for improving self-measurement adherence and overcoming the challenges related to moving from the supervised clinical setting to the unsupervised home setting. Also, we found that the interviewed patients were willing to accept more advanced monitoring systems and increased surveillance, as long as it is only used during self-measurement periods and is proportional to the severity of the disease.

\section{ACKNOWLEDGMENT}

Thanks to all participants and to Torben Lund Jensen, Heidi Fosborg Skovfoged, and Rasmus Elm Sørensen at the Electronics and Mechanics Workshop.

\section{REFERENCES}

[1] T.G. Pickering, W.B. White, T.D. Giles, H.R. Black, J.L. Izzo, B.J. Materson, S. Oparil and M.A. Weber, "When and how to use self (home) and ambulatory blood pressure monitoring," J.Am.Soc.Hypertens., vol. 4, pp. 56-61, Mar-Apr. 2010.

[2] T.G. Pickering, N.H. Miller, G. Ogedegbe, L.R. Krakoff, N.T. Artinian, D. Goff, American Heart Association, American Society of Hypertension and Preventive Cardiovascular Nurses Association, "Call to action on use and reimbursement for home blood pressure monitoring: executive summary: a joint scientific statement from the
American Heart Association, American Society Of Hypertension, and Preventive Cardiovascular Nurses Association," Hypertension, vol. 52, pp. 1-9, Jul. 2008

[3] E. O'Brien, R. Asmar, L. Beilin, Y. Imai, J.M. Mallion, G. Mancia, T. Mengden, M. Myers, P. Padfield, P. Palatini, G. Parati, T. Pickering, J. Redon, J. Staessen, G. Stergiou, P. Verdecchia and European Society of Hypertension Working Group on Blood Pressure Monitoring, "European Society of Hypertension recommendations for conventional, ambulatory and home blood pressure measurement," J.Hypertens., vol. 21, pp. 821-848, May. 2003.

[4] T.G. Pickering, "Ambulatory monitoring and blood pressure variability", Science Press, 1991,

[5] S.D. Pierdomenico, M. Di Nicola, A.L. Esposito, R. Di Mascio, E. Ballone, D. Lapenna and F. Cuccurullo, "Prognostic value of different indices of blood pressure variability in hypertensive patients," Am.J.Hypertens., vol. 22, pp. 842-847, Aug. 2009.

[6] S. Wagner, T.S. Toftegaard and O.W. Bertelsen, "Challenges in Blood Pressure Self-Measurement," International Journal of Telemedicine and Applications, 2012.

[7] S. Wagner, T. Toftegaard and O. Bertelsen, "Context Assessment during Blood Pressure Self-measurement Utilizing the Sensor Chair," Ambient Intelligence, pp. 295-299, 2011.

[8] Jacob Jørvang, Karina Kusk, Ditte B. Nielsen, Troels Thylstrup, Christian Fischer Pedersen and Stefan Wagner. Reliable Unsupervised Home Blood Pressure Self-Measurement with a Focus on Time-to-Rest using Sensor Fusion. Proceedings of the 5th Danish Conference on Pervasive Healthcare Engineering; (in press), 2012.

[9] S. Wagner, N.H. Rasmussen, P. Ahrendt, T.S. Toftegaard and O. Bertelsen, "Context classification during blood pressure selfmeasurement using the sensor seat and the audio classification device", in Proceedings of the 6th International Conference on Pervasive Computing Technologies for Healthcare (PervasiveHealth), pp. 201-202, 2012.

[10] S. Wagner, T.S. Toftegaard and O.W. Bertelsen, "The AdherenceStrategy Engineering Framework," In Preperation for Methods of Information in Medicine, 2012.

[11] G. Demiris. "Smart homes and ambient assisted living in an aging society. New opportunities and challenges for biomedical informatics". Methods Inf Med 2008;47(1):56-57.

[12] K.A. Johnson, D.J. Partsch, L.L. Rippole, D.M. McVey. "Reliability of self-reported blood pressure measurements". Arch Intern Med 1999 Dec 13-27;159(22):2689-2693.

[13] T. Mengden, R.M. Hernandez Medina, B. Beltran, E. Alvarez, K. Kraft, H. Vetter. "Reliability of reporting self-measured blood pressure values by hypertensive patients". Am J Hypertens 1998 Dec;11(12):1413-1417.

[14] M.G. Myers. "Self-measurement of blood pressure at home: The potential for reporting bias". Blood Press Monit 1998;3 (SUPPL. 1):S19-S22.

[15] W.P. Santamore, C.J. Homko, A. Kashem, T.R. McConnell, F.J Menapace, A.A. Bove. Accuracy of blood pressure measurements transmitted through a telemedicine system in underserved populations. Telemed J E Health 2008 May;14(4):333-338.

[16] S. Wagner, C.H. Kamper, T.S. Toftegaard O.W. Bertelsen "Blood Pressure Self-Measurement in the Obstetric Waiting Room". Submitted for Telemed J E Health, 2013.

[17] S. Wagner, N.H. Buus, B. Jespersen, T.S. Toftegaard, O.W Bertelsen. "Measurement Adherence in the Blood Pressure SelfMeasurement Room". Submitted for Telemed J E Health, 2013.

[18] A. Copetti, O. Loques, J.C.B. Leite, T. P. C. Barbosa and A. C. L. da Nobrega. "Intelligent context-aware monitoring of hypertensive patients." Proceedings of the 3rd International Conference on Pervasive Computing Technologies for Healthcare (PervasiveHealth). IEEE, 2009.

[19] LT D'Angelo, M. Lohmann and TC Lueth. "A new device for motionaware ambulatory blood pressure measurement". Proceedings of the 5th International Conference on Pervasive Computing Technologies for Healthcare (PervasiveHealth), IEEE, 2011. 